\title{
LAS EXPOSICIONES CIENTÍFICAS ESCOLARES Y SU CONTRIBUCIÓN EN EL ÁMBITO AFECTIVO DE LOS ALUMNOS PARTICIPANTES
}

\author{
Oliva, J. M. ${ }^{1.2}$; MAtos, J. ${ }^{1}$; Bueno, E. ${ }^{1}$; Bonat, M. ${ }^{1}$; Domínguez, J. ${ }^{1}$; V́́zquez, A. ${ }^{3}$ y ACEVedo, J.A. ${ }^{4}$ \\ ${ }^{1}$ Asociación de Profesores Amigos de la Ciencia EUREKA. Cádiz \\ ${ }^{2}$ Centro de Profesorado de Cádiz. Consejería de Educación y Ciencia de la Junta de Andalucía. Cádiz \\ ${ }^{3}$ Departamento de Inspección de Educación. Conselleria d’Educació i Cultura. Palma de Mallorca \\ ${ }^{4}$ Inspección de Educación. Consejería de Educación y Ciencia de la Junta de Andalucía. Huelva \\ Centro de Profesorado de Cádiz. C/Nueva de Puntales s/n. 11011. Cádiz \\ jmoliva@cepcadiz.com
}

\begin{abstract}
Resumen. En este trabajo se analiza la incidencia en el ámbito afectivo de los alumnos de «La Ciencia Divertida», una experiencia innovadora consistente en la planificación, construcción y realización de una exposición científica interactiva a cargo de grupos de alumnos y profesores. Los resultados obtenidos indican un alto grado de implicación y motivación del alumnado que diseñó los módulos y una incidencia positiva de la actividad en la autoestima y desarrollo personal de los alumnos. También sugieren una mejora de la convivencia y el clima de relaciones personales alumno-alumno y alumno-profesor, y en la motivación y satisfacción personal de los alumnos hacia la propia experiencia y la ciencia en general. Sin embargo, no se aprecian grandes logros respecto a la transferencia al aula de las actitudes positivas generadas hacia la experiencia desarrollada y la propia ciencia, aunque sí respecto al clima de relaciones personales.
\end{abstract}

Palabras clave. Actitudes hacia la ciencia, enseñanza formal, enseñanza no formal, exposición científica, museo de ciencias, variables afectivas.

Summary. In this paper, the influence of «The Amusing Science» on the students' attitudes is evaluated. This innovative experience includes the design, construction and assembly of a scientific interactive exposition made up by students and teachers. The results show high rates of students' implication and motivation and a positive influence in their self-steem and personal development. They also suggest an improvement of the interpersonal relationships among students and among students and teachers, as well as of the students' satisfaction to the experience and to Science. The improved interpersonal relationships were translated into science classroom without stating the positive attitudes.

Keywords. Affective variables, formal teaching, non-formal teaching, science museum, science attitudes, scientific exposition.

\section{PLANTEAMIENTO DEL PROBLEMA}

Uno de los problemas que más preocupa hoy día al profesorado de ciencias de la educación secundaria es el de la motivación de sus alumnos hacia el estudio. Esta percepción no está exenta de fundamento, como lo demuestran los abundantes estudios sobre actitudes realizados desde hace dos décadas (Yager y Yager, 1985; Vázquez y Manassero, 1995; Ramsden, 1998).

En efecto, las reformas a las que se han ido incorporando los países occidentales han traído como positivo la extensión de la etapa obligatoria de la educación hasta edades más tardías. Pero, con ello, ha surgido también un alumnado más diverso al que había antes en las escuelas, entre el que no es difícil encontrar un número importante con un bajo nivel de autoestima y escasa inclinación hacia al estudio, en ocasiones incluso con graves problemas afectivos y familiares. Ello, unido a prácticas tradicionales de enseñanza escasamente motivadoras, muy desligadas del componente experimental de la ciencia, suele conducir a climas de aula desconcertantes para el profesor y a problemas más o menos graves de disciplina. 
Sin embargo, la investigación en didáctica de las ciencias ha incidido mucho más en los procesos cognitivos que en los de tipo emotivo a la hora de avalar sus avances (Oliva et al., 2002). Por ejemplo, aunque problemas como el del cambio conceptual han sido analizados con detenimiento, se han desarrollado pocos estudios sobre la dimensión afectiva. Como Watts y Alsop (2000) ponían de manifiesto con ocasión de un editorial del International Journal of Science Education, esto pone al descubierto una importante carencia en nuestra área de conocimientos y sugiere la necesidad de mayor investigación en ese otro terreno. De hecho, los ámbitos de aprendizaje afectivos son frecuentemente relegados a un segundo plano en los programas educativos y de investigación, probablemente porque el aprendizaje afectivo es mucho menos conocido (Meredith, Fortner y Mullins, 1997) y también más difícil de evaluar y encuadrar teóricamente (McLeod, 1990; Gómez-Chacón, 1998). Pese a su larga tradición psicoeducativa, donde hay muchas aportaciones, podría decirse que los estudios sobre el papel de los aspectos afectivos se encuentran en una fase incipiente en la didáctica de las ciencias, lo que, sin duda, constituye un obstáculo importante a la hora de intentar fundamentar posiciones en este ámbito y también para elaborar propuestas didácticas concretas que tengan en cuenta esta dimensión del aprendizaje en la enseñanza de las ciencias.

Desde nuestro punto de vista, el aprendizaje y desarrollo en el ámbito afectivo constituyen en sí mismos un problema importante que debería ser objeto de mayor atención en la enseñanza de las ciencias (Oliva et al., 2002). Pero, además, hoy sabemos que los procesos cognitivos están profundamente influidos por sentimientos y emociones (Reid y Hodson, 1989), de forma que existe una relación profunda entre las variables emotivas y los procesos de cambio conceptual (Pintrich, Marx y Boyle, 1993). Como señalan los últimos autores citados, no es posible profundizar en la cognición del alumno solamente desde la exclusiva racionalidad de un frío cambio conceptual, sin tener en cuenta sus intereses, sentimientos y autopercepciones como aprendices.

En este trabajo se describen los resultados de una experiencia de innovación educativa realizada con grupos de escolares y profesores de educación secundaria, tendente a incidir especialmente en los ámbitos afectivo y actitudinal del aprendizaje (Oliva y Matos, 1999a, 1999b; Matos et al., 2001). La experiencia consistió en el diseño y puesta en marcha de una exposición científica interactiva, un museo de ciencia escolar, que tenía la peculiaridad de que la fabricación de sus módulos y el proceso de su enseñanza al público fue encomendada a grupos de escolares dirigidos por sus propios profesores. Aunque en la experiencia evaluamos distintas facetas relativas al alumnado y al profesorado participantes, este artículo se centra solamente en la repercusión de la misma en la dimensión afectiva de los alumnos.

\section{VARIABLES AFECTIVAS Y APRENDIZAJE DE LAS CIENCIAS}

La didáctica de las ciencias ha vivido frecuentemente de espaldas a muchos desarrollos de la investigación psico- educativa general, redescubriendo viejos logros de ésta a medida que se plantean aparentes nuevos problemas. Éste puede ser el caso de la investigación sobre las variables afectivas y el aprendizaje, que es muy extensa para ser siquiera resumida brevemente aquí. Incluso la referente a las variables puramente motivacionales es sumamente amplia y tiene desarrollos muy diversos, desde los planteamientos más mecanicistas (basados en los conceptos de placer y dolor) hasta las más actuales teorías cognitivas de la motivación, que asumen la influencia intrínseca de las necesidades sociales, los pensamientos y las emociones en la conducta humana, en general, y en las conductas de logro como el aprendizaje, en particular (McClelland, 1961; Weiner, 1992). En este último marco teórico, variables afectivas como las relacionadas con expectativas, valores, atribuciones y emociones juegan un papel esencial en el aprendizaje escolar (Manassero y Vázquez, 1998, 2001).

En el ámbito concreto de la didáctica de las ciencias, algunos de los autores pioneros de los modelos de cambio conceptual advierten que los estudiantes que mantienen mayor confianza en su habilidad para aprender ciencias y también aquéllos que valoran mejor la ciencia son los que consiguen llevar a cabo un aprendizaje más adecuado para el cambio conceptual (Strike y Posner, 1992). En consonancia con ello, Reid y Hodson (1989) señalan que sólo en la medida en que, como profesores, centremos la atención en las necesidades personales y afectivas de los alumnos, podremos comenzar a elevar los niveles de rendimiento en el ámbito académico. En la misma línea, Laukenmann, Grob y Rhönnek (1999) apuntan hacia la motivación por el aprendizaje, el clima social y el autoconcepto en la materia específica como factores clave que condicionan el aprendizaje en el aula.

Otro referente importante de esta investigación lo encontramos en el ya citado trabajo de Pintrich y otros (1993). En el mismo se señala que, cuando los alumnos tienen una motivación intrínseca por el contenido del aprendizaje, es más probable que éstos se involucren más profundamente en la tarea y, con ello, en estrategias metacognitivas de autorregulación. En tales circunstancias, señalan Pintrich y otros (1993), será más probable también que se den las condiciones para el cambio conceptual previstas por Posner y otros (1982).

Pero el tipo de motivación que muestra el alumno no es algo arbitrario, sino que depende, a su vez, del contexto escolar y del tipo de tarea o actividad de aula puesta en juego. En este sentido, la investigación desarrollada durante los últimos años ha llegado a delimitar algunos de los rasgos de las tareas que pueden contribuir a un desarrollo de la motivación intrínseca en el proceso de aprendizaje (Wheatley, 1991; Pintrich et al., 1993; Baird, 1997). Entre esas condiciones encontramos:

- Que las tareas sean verdaderamente significativas para los alumnos, es decir, que encajen en el perfil de intereses y conocimientos que poseen.

- Que sean relevantes para su vida diaria, lo que reportará una mayor familiaridad y aplicabilidad. 
- Que se presenten de un modo desafiante, como una curiosidad, una paradoja o con un factor sorpresa.

- Que sean novedosas, combinando tareas de distinto tipo y evitando la monotonía y la rutina.

- Que inviten a los alumnos a tomar decisiones, más que a seguir recetas o protocolos preestablecidos que se tengan que aprender de manera rutinaria.

Entre las tareas que Pintrich y otros (1993) citan como típicas de este perfil encontramos las actividades abiertas $y$, entre ellas, los experimentos de ciencias que recurren a métodos de descubrimiento e indagación (Von Secker y Lissitz, 1999; Lynch y Zenchak, 2002), por cuanto disminuyen el papel autoritario del profesor y la forma en la que los alumnos son evaluados.

Además, Pintrich y otros (1993) muestran la importancia de la autoestima en el aprendizaje y señalan algunas propuestas concretas para el planteamiento de actividades que desarrollen este factor en el alumno. Entre dichas propuestas mencionan la de propiciar que los alumnos aprecien cómo algunas tareas que inicialmente perciben de difícil resolución, luego son capaces de controlarlas y resolverlas adecuadamente, o la de apreciar cómo otros alumnos pueden llegar a topar con las mismas dificultades con las que se han encontrado ellos al enfrentarse a una tarea semejante. En contraposición, la enseñanza de las ciencias de tipo convencional recurre poco a la curiosidad natural de los estudiantes y les deja con la impresión de que son incapaces de comprender la ciencia (AAAS, 1990; Lynch y Zenchak, 2002).

Se desprende de todo lo anterior que la motivación no sólo es un requisito previo al aprendizaje sino también una consecuencia de la enseñanza (Pozo y Gómez-Crespo, 1997), y que la actitud del alumno y el clima del aula son un producto natural derivado de lecciones interesantes y de una saludable relación entre maestro y alumno, resultado de una buena práctica educativa (Ausubel, Novak y Hanesian, 1989). En definitiva, tanto la motivación como la propia disciplina en el aula dependen, en gran parte, de la implicación de los alumnos en tareas que les resulten relevantes, de la percepción de los progresos en las mismas y de la valoración positiva que ese trabajo recibe, todo lo cual contribuye a generar un ambiente de trabajo ordenado y distendido y, en definitiva, a un cambio actitudinal (Gil et al., 1991).

De ahí quizás se desprende la desmotivación que suele apreciarse en determinadas aulas, en contraste con el entusiasmo que los alumnos, y también los profesores, pueden llegar a mantener en actividades de ciencias llevadas a cabo en contextos no formales fuera del aula (Oliva y Matos, 1999a, 1999b; Crespo y Hernández, 2000). En efecto, no es difícil ver cómo algunos de los alumnos y alumnas que consideramos de peor rendimiento o poco motivados nos sorprenden favorablemente cuando se involucran voluntariamente en este tipo de tareas, en ocasiones con mayor pasión y éxito incluso que aquéllos otros que se suponen más aventajados (Oliva y Matos, 1999a). La confección, por ejemplo, de murales con motivo de alguna semana o jornada científica organizada en una escuela, la afición personal a determinados temas de ciencia como la zoología, la ecología o la astronomía, o bien las visitas extraescolares a museos o centros de ciencias, etc. son solamente algunos ejemplos de facetas extraacadémicas por las que los alumnos suelen sentir una cierta inclinación a pesar de mantener actitudes y comportamientos negativos en las clases de ciencias.

\section{ENSEÑANZA DE LAS CIENCIAS Y APRENDI- ZAJE EN AMBIENTES NO FORMALES}

Aunque el número de trabajos publicados sobre el aprendizaje de las ciencias en contextos no formales empieza a ser elevado, y aumenta día a día, se han publicado muy pocos estudios sobre experiencias que canalicen esos procesos de aprendizaje desde una perspectiva académica (Lucas, 1991), y ello a pesar de que los conocimientos adquiridos informalmente podrían ser útiles desde la perspectiva de la enseñanza de las ciencias en el aula (Lucas, McManus y Thomas; 1986; Tamir, 1990; AAVV, 1991; Rix y McSorley, 1999).

Así, por un lado, Schibeci y Riley (1986) examinaron experimentalmente el impacto del entorno de aprendizaje informal dentro del hogar sobre el rendimiento de los alumnos en ciencias obteniendo resultados positivos. Por otro lado, Tamir (1990) ha mostrado que la participación de los alumnos en actividades de ciencias extraescolares estaba fuertemente relacionada con su compromiso hacia las ciencias y el aprendizaje de las ciencias. Además, Gerber, Cavallo y Marek (2001) han aportado datos acerca de los efectos positivos de los entornos de aprendizaje informal sobre las habilidades de razonamiento científico de los alumnos. Por último, Jones (1997) ha mostrado su utilidad como recurso para afrontar el problema de la atención a la diversidad en la clase de ciencias.

Con estos datos en la mano, cabe preguntarse: ¿qué diferencias existen entre el modo de aprender ciencias en las escuelas y el modo de aprender que se promueve desde estos entornos informales? La respuesta a esta pregunta nos parece clave, ya que sería de gran interés, para el entorno escolar, aproximar sus métodos de enseñanza de las ciencias a los que promueven esos otros contextos informales, donde los alumnos parecen estar mucho más motivados. En un intento de responder a esta pregunta, podemos adoptar el guión que ofrece Wellington (1991) para diferenciar las características de los entornos formales y no formales (Tabla I). 
Tabla I

Aprendizaje científico en ambientes formales y no formales (Wellington, 1991).

\begin{tabular}{|l|l|}
\hline \multicolumn{1}{|c|}{ Aprendizaje informal } & \multicolumn{1}{c|}{ Aprendizaje formal } \\
\hline Voluntario & Obligatorio \\
\hline Fortuito, desestructurado y no secuenciado & Estimulado, estructurado y secuenciado \\
\hline No evaluado ni certificado & Evaluado y certificado \\
\hline Guiado por el que aprende y centrado en el que aprende & Guiado por el profesor y centrado en el profesor \\
\hline No planificado & Planificado \\
\hline Muchos efectos indeseables & Muy pocos efectos indeseables \\
\hline Centrado en aspectos sociales & Menos centrado en aspectos sociales \\
\hline De poca «utilidad»* & De gran «utilidad»* \\
\hline No dirigido ni reglado & Reglado y dirigido (controlado) \\
\hline
\end{tabular}

* Se refiere aquí al partido que el sujeto pueda extraer del mismo.

Como puede verse, al menos bajo la forma en la que Wellington lo plantea, ambos perfiles presentan rasgos netamente contrapuestos; de ahí que sea difícil adaptar el uno al otro como en principio estaríamos tentados a realizar. En vez de ello, lo más adecuado sería considerar a ambos como realidades distintas, aunque interconectables, utilizando el primero como recurso didáctico del segundo (Oliva y Matos, 2000). De cualquier modo, lo que sí parece claro es la necesidad de interrelacionar ambos tipos de aprendizajes, entre otras razones para evitar una compartimentación de las adquisiciones logradas por una u otra vía o el planteamiento de conflictos entre ambas (Wellington, 1991).

Lucas (1991) destaca el ejemplo de las visitas a los museos de ciencias como un caso emblemático en el ámbito de las posibles relaciones entre el aprendizaje de las ciencias dentro y fuera del aula. Tomando en cuenta dicho nexo, y desde una óptica afectiva como la que se pretende adoptar en este trabajo, puede resultar pertinente citar aquí los trabajos de Stevenson (1991) o Medved y Oatley (2000), en los que se ponen de relieve el papel de esas visitas a la hora de despertar actitudes emotivas en las personas, como sorpresa, fascinación, diversión, curiosidad, etc. En la misma línea apunta el trabajo de Russell (1990), en el que se resalta la aportación de los centros de ciencias sobre el componente afectivo del aprendizaje, especialmente para cambiar los intereses, la curiosidad, las ganas de aprender, mejorar la autoconfianza, el gusto por la ciencia e, incluso, el deseo de convertirse en científico. Por su parte, Semper (1990) señala que la atmósfera informal que se crea en un centro de ciencias propicia la interacción espontánea con amigos, familia y profesores, lo cual ayuda a consolidar el aprendizaje. Por ejemplo, los padres suelen mostrar con sus hijos una actitud de acompañamiento muy positiva durante las visitas, animándoles a observar y a actuar con el módulo, ayudándoles en sus requerimientos y escuchándoles y respondiéndoles a sus comentarios (Benlloch y Williams, 1998).
Especial mención merece el estudio de Rix y McSorley (1999), en el que se exploró el papel en el aprendizaje de los alumnos de los «minicentros» de ciencias interactivos que empiezan a desarrollarse en algunas escuelas con fines didácticos. Su trabajo muestra aportaciones positivas en distintos ámbitos, entre los que destaca el de las actitudes positivas hacia la ciencia. Como señalan los autores, el entusiasmo desarrollado en los niños proporciona luego al profesor de ciencias una oportunidad única para sacar partido a las experiencias interactivas en un contexto más académico. En la misma dirección cabe citar el trabajo de Lucas (2000), en el que se justifica y detalla la creación por los alumnos de un «minicentro» de ciencias en su propia aula, como parte de la preparación de una visita a un centro de ciencias.

Por otra parte, es conveniente aclarar y relacionar los conceptos de educación no formal, educación en valores y enseñanza mediante proyectos, porque ayudarán a precisar el marco de la experiencia realizada. La educación en valores hace referencia a la propuesta de conseguir objetivos educativos del ámbito socioafectivo, que en nuestro sistema educativo se han formulado como actitudes, valores y normas y, por tanto, está incluida en la educación formal, aunque sea poco frecuente en la realidad actual de la enseñanza de las ciencias. La enseñanza mediante proyectos es una metodología educativa bien conocida en la didáctica general, que consiste en la realización por los estudiantes de una tarea de mayor amplitud y participación que las actividades usuales de aula, generalmente con un plazo temporal y de recursos que supera el contexto habitual del aula. Ambas constituyen actividades propias de la educación formal, aunque, en el marco de la enseñanza de las ciencias, hayan sido tan poco utilizadas que muchos profesores las pueden considerar prácticas no formales; la mirada a los museos y las ferias de la ciencia nos sugieren la relevancia de ambas y la experiencia desarrollada conjuga ambos elementos y ratifica su idoneidad educativa. 
Como conclusión de todo lo anterior, nuestra hipótesis es que la enseñanza formal debería retomar estos aspectos informales de las ciencias en las actividades escolares que se llevan a cabo dentro y fuera del aula de ciencias, ya que ello repercutiría sustancialmente en la motivación y actitudes de los alumnos y, en consecuencia, también en la motivación y actuación de sus profesores. Concretamente, creemos que el acercamiento a la escuela de ciertos ambientes más lúdicos de aprendizaje, como los que ofrecen los centros de ciencias a través de fenómenos y experiencias atractivas, podría aportar un contexto sensible al panorama apuntado, ayudando a complementar, enriquecer y mejorar la educación científica que se suele ofrecer en las situaciones más formales del aula (Griffin, 1998).

La novedad de este trabajo con respecto a otros que analizan el papel en el aprendizaje de las experiencias interactivas en los centros de ciencias (Medved y Oatley, 2000; Russell, 1990; Stevenson, 1991), incluso dentro de ambientes escolares (Rix y McSorley, 1999), es que, en este caso, los alumnos se convirtieron en los protagonistas de la experiencia, siendo ellos los que construyeron los módulos y los que, así mismo, realizaron la función de animadores y de atención al público en el desarrollo de la exposición (Matos et al., 2001; Oliva et al., 2002).

\section{DESCRIPCIÓN DE LA EXPERIENCIA}

Las pretensiones que han orientado la experiencia podrían resumirse en torno a los siguientes puntos:

1) Implicar al alumnado en el proceso de enseñanzaaprendizaje fomentando actitudes positivas hacia la ciencia y su aprendizaje.

2) Contribuir al desarrollo de la creatividad y de actitudes y procedimientos científicos asociados a este tipo de actividad.

3) Crear una actitud de colaboración, participación y responsabilidad en el alumnado.

4) Proporcionar un entorno en el que se desarrolle favorablemente el clima de relaciones humanas.

5) Hacer protagonistas a los alumnos y mejorar su autoestima, a través de una experiencia repleta de obstáculos y dificultades, pero que al final pudiera aportar la satisfacción de ver reconocido su trabajo.

6) La posibilidad de transferir ese clima de trabajo y las habilidades adquiridas al aprendizaje formal de las aulas.

La exposición contó con un conjunto de módulos de experimentos interactivos, seleccionados por su significación científica y por resultar llamativos y, en la medida de lo posible, sorprendentes, como es habitual en los museos y centros de ciencia y tecnología. La primera propuesta de módulos fue realizada por una comisión organizadora, integrada entre otras personas por los cinco primeros autores de la investigación. Posteriormente, el resto de profesores participantes tuvo la oportunidad de aumentar la lista con módulos adicionales hasta completar un total de más de sesenta posibles.

Posteriormente se pasó a la fase de asignación de los módulos a cada centro, para lo cual se tuvo en cuenta, entre otros criterios, las preferencias de los profesores implicados. Cada centro se responsabilizó de dos o tres módulos, lo que supuso el desarrollo de un total de cuarenta y cinco módulos de los más de sesenta preseleccionados. Éstos fueron diseñados y fabricados por los propios alumnos distribuidos en pequeños grupos. En cada caso, el trabajo fue realizado como una pequeña investigación (Gil y Payá, 1988) dirigida y coordinada por el profesor de ciencias. La construcción del módulo se enfocó como la resolución de un problema abierto de carácter práctico (Carrascosa, 1995; Garret, 1995), desarrollándose en un ambiente a mitad de camino entre los aprendizajes escolar e informal e inspirándose en las corrientes de ciencia recreativa. Se trataba de poner los conocimientos teóricos y procedimentales al servicio de la práctica experimental (Sèré, 2002), a la vez que ésta sirve también como recurso para favorecer la evolución conceptual de los alumnos sobre los fenómenos que abordaban.

Se marcaron dos objetivos fundamentales: primero, el diseño, fabricación y puesta a punto de distintos módulos interactivos, que se llevó a cabo desde noviembre hasta abril del curso 1999-2000; y, segundo, su inclusión en una exposición que, bajo el título de «La Ciencia Divertida», recorrió de forma itinerante cinco poblaciones de la bahía de Cádiz de abril a junio del mismo curso, a razón de una semana en cada una, aproximadamente. Como lugar para la exposición pública se eligieron lugares céntricos de las localidades, de fácil acceso para los visitantes, más concretamente locales dedicados habitualmente a exposiciones de arte o a actividades juveniles. Los alumnos, junto a sus profesores, se responsabilizaron del traslado y montaje de la exposición, que se realizó durante los fines de semana. Así mismo, todos ellos se encargaron de la atención al público visitante durante el tiempo de apertura al público, que fue de lunes a viernes en horario de mañana y tarde.

La mayor parte del tiempo invertido en la construcción de los módulos se llevó a cabo por las tardes en horas no lectivas, unas veces dentro y otras fuera del recinto escolar. No obstante, con bastante frecuencia, los profesores implicados en la experiencia intentaron además relacionar dicho proceso con el currículo escolar, dedicando parte de sus clases de ciencias a dicha tarea. Incluso, en determinados casos, hubo coordinación con el profesor de tecnología para que éste dedicase parte de sus clases a que los alumnos implicados construyesen las piezas más complejas de los módulos.

Como culminación del trabajo realizado, los alumnos participaron en unas jornadas finales intercentros, de una mañana de duración, en la que se formaron como monitores «expertos» para la totalidad de módulos de la exposición. Asumieron, de esta forma, todos ellos, la tarea de vigilancia y explicación al público, al me- 
nos durante un día completo (mañana y tarde). Esta inversión de horario lectivo por parte de alumnos y profesores fue posible gracias a la canalización de la exposición como actividad extraescolar complementaria programada en los centros por los distintos departamentos participantes.

La mayoría de materiales utilizados en la construcción de los módulos fueron económicos y de fácil adquisición, con una filosofía muy próxima a la de los clásicos manuales de la UNESCO de trabajo experimental a partir de materiales de bajo coste. Aunque todos ellos resultaban susceptibles de mejora en cuanto a su diseño y calidad, hay que hacer constar que, en la práctica, han «soportado» la manipulación de un elevado número de personas con un gasto mínimo de mantenimiento.

El diseño de los módulos supuso una ocasión inmejorable para poner en evidencia algunas relaciones existentes entre la ciencia y la tecnología (Maiztegui et al., 2002; Valdés et al., 2002; Acevedo y Vázquez, 2003), siempre en un plano de igualdad entre ambas y respetando sus características propias (Acevedo, 1995), mientras que la exposición al público fue una rica experiencia respecto a las relaciones ciencia-sociedad, sobre todo en lo referente a la divulgación y aproximación de la ciencia a la ciudadanía por los propios alumnos; esto es, para mejorar la comprensión pública de la ciencia. En conjunto, «La Ciencia Divertida» propició también diversas estrategias de enseñanza-aprendizaje recomendadas por la educación CTS (ciencia-tecnología-sociedad), por lo cual puede considerarse una aportación interesante para la alfabetización científica y tecnológica que tiene en cuenta determinadas orientaciones de los enfoques CTS (Acevedo, 1996; Acevedo, Vázquez y Manassero, 2003; Acevedo-Pineda, en línea) y está en sintonía con criterios de relevancia de la ciencia escolar más adecuados a las finalidades de la educación científica para todas las personas (Acevedo, 2004).

En la experiencia se han visto involucrados alrededor de 500 alumnos y 70 profesores procedentes de 19 centros escolares de secundaria (públicos y concertados) de distintas localidades de la comarca de la Bahía de Cádiz. No obstante, sólo alrededor de la mitad de los mismos se vieron implicados directamente en el diseño de los módulos y en los aspectos organizativos de la muestra. El resto ha intervenido sólo parcialmente cubriendo aspectos concretos, como la colaboración ocasional en el diseño de los módulos o la vigilancia y supervisión durante algún día de la exposición. Tanto el alumnado como el profesorado integrado en la experiencia se prestaron de forma voluntaria a participar en ella, en ambos casos con una participación equilibrada en el género, y en el primero abarcando un amplio espectro de perfiles respecto a niveles académicos y estatus socioeconómico.

Se estima que el número total de visitantes a la exposición ha superado los 25.000 asistentes. Todo el proceso de diseño, organización, realización y evaluación de la experiencia estuvo coordinado por los dos primeros autores del trabajo, dentro del contexto más amplio de la comisión general organizadora mencionada más arriba.

\section{METODOLOGÍA DE LA EVALUACIÓN}

El estudio realizado a partir de los datos obtenidos ha sido de corte interpretativo. Si bien se ha combinado información tanto de tipo textual como numérica, el tipo de análisis realizado ha seguido los cánones habituales de la evaluación cualitativa. En ella ha participado una muestra representativa de los alumnos y profesores que intervinieron de una forma más directa en la experiencia.

Los instrumentos utilizados tenían por objeto detectar cómo había incidido la experiencia en distintos aspectos relativos al aprendizaje de los alumnos y el desarrollo profesional de los profesores participantes (Matos et al., 2001). Sin embargo, en este trabajo nos referiremos solamente a aquellos aspectos relativos a las opiniones y valoraciones que formularon alumnos y profesores sobre la incidencia de la experiencia en la dimensión afectiva de los estudiantes participantes.

De una parte, contamos con la información procedente de un cuestionario administrado tras finalizar la experiencia a profesores y alumnos, ambos muy similares aunque con pequeñas variantes para adaptar las preguntas y el lenguaje utilizado a cada colectivo concreto. Dicho cuestionario estaba integrado por un ítem abierto, dirigido a expresar comentarios sobre los aspectos positivos y negativos más relevantes de la experiencia, y catorce ítems más, formulados en formato cerrado con cinco opciones que recorrían distintos grados de acuerdo o desacuerdo respecto a las diferentes cuestiones propuestas. Estos ítems fueron seleccionados en función de su capacidad potencial para valorar determinados aspectos significativos de la experiencia que se quería evaluar, y fueron objeto de un proceso previo de validación de contenido por un equipo de ocho jueces entre los que figuraban los cinco primeros autores del trabajo. Aquellos ítems propuestos en primera instancia que no superaron dicho filtro fueron modificados o eliminados por consenso. La elección y redacción de los ítems se ajustó a los criterios y recomendaciones que se suelen proponer para los cuestionarios utilizados en la investigación cualitativa (Rodríguez et al., 1996).

De esos catorce ítems, en este estudio se consideran sólo seis, que son aquéllos más relacionados con las dimensiones que definiremos al presentar los resultados de la experiencia, centrándonos aquí sólo en la vertiente socioafectiva. En el anexo se recogen, como ejemplos, tres ítems del cuestionario. Estos ítems pretenden evaluar la repercusión de la experiencia en el clima de relaciones personales (profesor-alumno y alumno-alumno) y en las actitudes de los alumnos hacia las ciencias y hacia la propia experiencia en la que participaron.

El cuestionario fue administrado a una muestra representativa del alumnado ( $\mathrm{N}=170 ; 73$ chicos y 97 chicas $)$ y el profesorado $(\mathrm{N}=29)$ que participó de una forma más activa en la experiencia, procedentes de catorce centros de educación secundaria. La composición de la muestra de alumnos se desglosa por cursos en la tabla I.

Los resultados aportados por los distintos ítems fueron valorados por separado, ítem a ítem, dado que cada uno 
de ellos tenía una finalidad diferente y no cabía esperar la existencia de un factor o constructo común en el que pudiesen englobarse todos ellos.

Además, se hicieron entrevistas individuales semiestructuradas a una muestra reducida de alumnos participantes procedentes de dos centros distintos $(\mathrm{N}=9)$. Dicha entrevista se realizó con posterioridad a la administración del cuestionario y su propósito era esencialmente comprobar la congruencia de los resultados de aquél, sirviendo como elemento de triangulación. La distribución de alumnos por cursos se muestra también en la tabla II. Tras algunas tareas dirigidas a evaluar cómo había incidido la experiencia en sus concepciones y creencias sobre los fenómenos en los que trabajaron, se recogieron sus valoraciones en el plano afectivo. Las preguntas formuladas fueron del tipo: ¿Qué te ha parecido la experiencia? ¿Qué ha sido para ti lo mejor y lo peor de participar en ella? ¿Cómo crees que ha incidido en tu forma de ver la ciencia y en tu actitud y comportamiento en clase?

Las entrevistas fueron grabadas en audio y posteriormente transcritas a papel.

Tabla II

Distribución de alumnos encuestados según instrumento y nivel escolar.

\begin{tabular}{|c|c|c|}
\hline Curso & Cuestionario & Entrevista \\
\hline $2^{\circ} \mathrm{ESO}$ & - & 5 \\
\hline $3^{\circ} \mathrm{ESO}$ & 36 & - \\
\hline $4^{\circ} \mathrm{ESO}$ & 114 & 4 \\
\hline $1^{\circ}$ bachillerato/3 ${ }^{\circ} \mathrm{BUP}$ & 14 & - \\
\hline $2^{\circ}$ bachillerato/COU & 6 & - \\
\hline
\end{tabular}

Finalmente se tomaron, como fuente de datos, las memorias de seis grupos de trabajo de profesores que se constituyeron formalmente en torno al centro de profesorado de la zona, tomando como motivo la reflexión sobre la actividad en la que habían participado. Cada grupo estaba integrado por cuatro o cinco profesores, normalmente de la misma localidad y, en algunos casos, incluso del mismo instituto. Las memorias abordaban aspectos relacionados con la dinámica de trabajo seguida en el grupo, la información referente a la labor que realizaron con los alumnos y las valoraciones sobre el desarrollo de la experiencia.

Toda la información recopilada, tanto textual como numériça, fue inicialmente dividida en unidades de análisis. Éstas consistían en frases, párrafos de las memorias del profesorado o fragmentos de entrevistas, según la fuente utilizada, que tenían un significado propio y hacían alusión a un mismo aspecto; o bien, en su caso, en promedios de las puntuaciones de 1 a 5 si la información procedía de los ítems cerrados del cuestionario. Una vez efectuado dicho proceso, la información resultante fue filtrada, y se seleccionó sólo aquélla que respondiese a los propósitos de este estudio.

A pesar de la cantidad y variedad de datos recopilados, optamos por efectuar un análisis de tipo global o ecológico en lugar de recurrir a estudios separados para cada uno de los instrumentos y muestras. Por ello, independientemente de la fuente de procedencia, las diferentes unidades de análisis obtenidas fueron clasificadas de acuerdo con un mismo sistema de categorías, que se fue gestando poco a poco en paralelo al proceso de análisis de resultados.

\section{RESULTADOS}

Las unidades de análisis que se identificaron a partir de las distintas fuentes quedaron clasificadas definitivamente en torno a cuatro categorías diferentes, que fueron los siguientes:

a) participación e implicación del alumnado;

b) cambios de actitudes en los alumnos;

c) grado de autorrealización que ha promovido (autoestima y madurez personal);

d) contribución al clima de convivencia y de relaciones personales.

La mayor parte de la información obtenida de tipo textual valoraba positivamente la experiencia. Los escasos comentarios encontrados con tintes críticos se referían normalmente a la gestión, al mantenimiento o a las dificultades organizativas, dimensión que debería ocupar un capítulo aparte, dado que no está directamente relacionada con el objeto de estudio de este trabajo. Otros, más esporádicos, sí se referían a las categorías señaladas y, por ello, serán analizados en la exposición.

A continuación se comentarán los resultados obtenidos en cada uno de los ámbitos señalados, algunos de los cuales fueron ya adelantados en un estudio anterior (Oliva et al., 2002).

\section{Sobre el nivel de participación e implicación de los alumnos}

La participación e implicación del alumnado fue, sin duda, lo más valorado por alumnos y profesores, como lo demuestra el hecho de que fuera también lo más citado a la hora de efectuar un balance global de la experiencia. En general, se destaca el alto grado de intervención y compromiso mostrado por la mayoría de los participantes, así como el entusiasmo generado con dicha participación. Lo que a continuación se presenta no es sino una pequeña muestra de referencias encontradas en las consultas realizadas a los distintos estamentos que intervinieron en la evaluación:

«Lo mejor de todo ha sido comprobar el grado de participación e interés mostrado por los alumnos.» (Profesor) 
«El aspecto que más resaltaría es el de la participación de los alumnos en la realización y explicación de los módulos.» (Profesor)

«Yo diría que el éxito [...] ha tenido que ver con la implicación de los alumnos participantes.» (Grupo de trabajo de profesores núm. 1)

«Todo el mundo ha participado y puesto mucho interés.» (Alumna de $3^{\circ}$ de ESO)

«[...] la mayoría de los compañeros y compañeras que se comprometieron, cumplieron a la perfección.» (Alumno de $3^{\circ}$ de ESO)

Un aspecto digno de mención es el mecanismo a través del cual se generó normalmente dicha implicación. En un cierto número de casos, tanto alumnos como profesores describían dicho proceso aludiendo a un efecto «dominó» o de «bola de nieve», en el que el entusiasmo y las ganas de integrarse en la experiencia se «transmitía» $\mathrm{y}$ «contagiaba» de unos a otros, haciendo que cada vez fuera mayor el número de alumnos interesados en participar. De este modo, lo normal fue que, en los centros, el proyecto se iniciara a partir de un pequeño núcleo de alumnos y profesores, el cual, más tarde, fue creciendo e incorporando nuevos participantes a medida que el proyecto avanzaba:

«En mi centro, el proyecto se propuso inicialmente a un pequeño grupo de once alumnos de $3^{\circ}$ de ESO, que se prestaron voluntariamente a participar en el mismo. A medida que se iba desarrollando la experiencia, sus compañeros de clase [...] solicitaron su incorporación en el proyecto y, finalmente, se amplió a toda la clase.» (Grupo de trabajo de profesores núm. 2)

«Poco a poco parecía que el entusiasmo crecía y se transmitía a otros alumnos que en principio no parecían interesados en participar» (Profesor)

«Al principio estaba indeciso, no sabía si apuntarme o no [...] Pero luego los amigos me convencieron, ya que cuando [...] hablaban entre ellos parecían entusiasmados con la idea.» (Alumno de $4^{\circ}$ de ESO)

Otro aspecto interesante a destacar es que no todos los alumnos que mostraron más compromiso con la actividad fueron alumnos aventajados desde un punto de vista académico. Por el contrario, algunos de los alumnos que mostraron mayor entusiasmo y dominio de la situación fueron estudiantes que eran considerados como «malos» $\mathrm{o} \ll$ mediocres» por sus profesores:

«Algunos alumnos que jamás creí que podrían funcionar en una actividad como ésta, se implicaron como los que más.» (Profesora)

«Algunos alumnos que parecían apáticos en el aula, me sorprendieron favorablemente cuando los vi trabajar en la experiencia.» (Profesor)

«[Cita el nombre de un alumno], que es uno de los peores de la clase, resultó un manitas a la hora de construir el módulo.» (Profesora)

Como puede verse, el comportamiento de los alumnos rompía en algunos casos con los estándares que suelen servir para etiquetar a los alumnos en contextos más convencionales de aula. Es posible que en un clima de trabajo como el generado en la experiencia, más abierto, sin presiones y con una fuerte valoración del componente creativo, fuera capaz de estimular a esos alumnos «poco aventajados» y sacar lo mejor que hay en ellos.

No obstante, y aunque los aspectos positivos predominaron claramente en este ámbito, esporádicamente también detectamos algunos comentarios negativos. Las escasas referencias encontradas en tales términos aludían a un cierto desequilibrio a la hora de asumir las tareas por parte de algunos participantes, lo que se traducía en una cierta concentración de funciones en unos y en una mayor pasividad e inhibición en otros:

«La participación de los alumnos fue desigual.» (Profesor)

«Lo peor ha sido la falta de responsabilidad y colaboración de algunos compañeros.» (Alumno)

No obstante, este desequilibrio parece que se produjo sólo en casos aislados, a tenor del escaso número de referencias encontradas al respecto, muy localizadas en centros concretos. Por tanto, el balance global que puede hacerse en este ámbito es positivo, debido en gran parte al interés despertado por el propio contenido de la actividad, a través de la cual los alumnos parecieron desarrollar actitudes sumamente favorables.

\section{Sobre el cambio de actitudes en los alumnos}

Otro de los aspectos mejor valorados fue el de las actitudes despertadas en los alumnos hacia su participación en la «Ciencia Divertida». Por lo general, la actividad resultó bastante sugerente e interesante, de lo cual quedó constancia a través de las opiniones de alumnos y profesores. He aquí algunos comentarios provenientes de los cuestionarios administrados a ambas partes y un fragmento procedente de la entrevista realizada a una alumna de $2^{\circ}$ de ESO.

«Me ha parecido una actividad enormemente interesante y motivadora para los alumnos.» (Profesor)

«Sobre todo me ha parecido genial por la ilusión que ha originado en los alumnos.» (Profesora)

«Todo lo que puedo decir de la experiencia es positivo. Me ha agradado participar en ella y todo me ha parecido fantástico.» (Alumno de $3^{\circ}$ ESO)

Entrevistador: «¿Qué es lo que te ha parecido mejor de tu participación en La Ciencia Divertida?»

Alumna: «Pues todo [...] No sé. Todo me ha parecido interesante.»

Entrevistador: «Pero me imagino que podrías destacar algo, ya sea bueno o malo.»

Alumna: «La verdad es que todo ha estado muy bien [...] Pero vamos, sobre todo destacaría [...] montar los módulos, participar en la exposición [...] y explicar las cosas al público.»

No obstante, en ocasiones, algunas opiniones manifestaron también posiciones más críticas, sobre todo en lo relativo al deterioro del material y el tiempo que le tuvieron que dedicar: 
«Lo peor ha sido aguantar a la gente que rompía cosas.» (Alumno)

«Aunque ha valido la pena, la verdad es que el tiempo que le hemos tenido que dedicar ha sido demasiado.» (Alumno)

Por otra parte, quisimos también evaluar hasta qué punto la experiencia contribuyó a mejorar las actitudes de los alumnos hacia las ciencias. Los resultados obtenidos indican una incidencia positiva de «La Ciencia Divertida» en esta vertiente, como se aprecia en las opiniones que siguen, aportadas por los participantes:

«La experiencia ha servido para que los alumnos [...] desarrollen su creatividad, curiosidad y entusiasmo.» (Grupo de trabajo de profesores núm. 2)

«Los alumnos se lo han pasado bien, han aprendido y han cogido gusto por la ciencia.» (Profesora)

«Lo más importante para mí ha sido darme cuenta de que la Ciencia es amena y divertida.» (Alumno de $3^{\circ}$ de ESO)

«La experiencia ha fomentado mi interés por investigar.» (Alumno de $3^{\circ}$ de ESO)

«[La experiencia] ha servido para mejorar mi opinión sobre la ciencia.» (Alumna de $4^{\circ}$ de ESO)

Éstos son sólo algunos de los comentarios aportados, los cuales revelan una repercusión positiva de la experiencia en actitudes tales como el comportamiento creativo, el gusto por «hacer» ciencia y, en general, el interés por la misma. Desde un punto de vista cuantitativo, la figura 1 recoge los resultados de las respuestas aportadas por profesores y alumnos en cada uno de los cuatro ítems cerrados de los cuestionarios relacionados con esta dimensión.

Como se puede apreciar, las valoraciones de los profesores fueron algo superiores a las de los alumnos, si

Figura 1

Opiniones sobre la incidencia de la experiencia en las actitudes hacia las ciencias de los alumnos participantes.

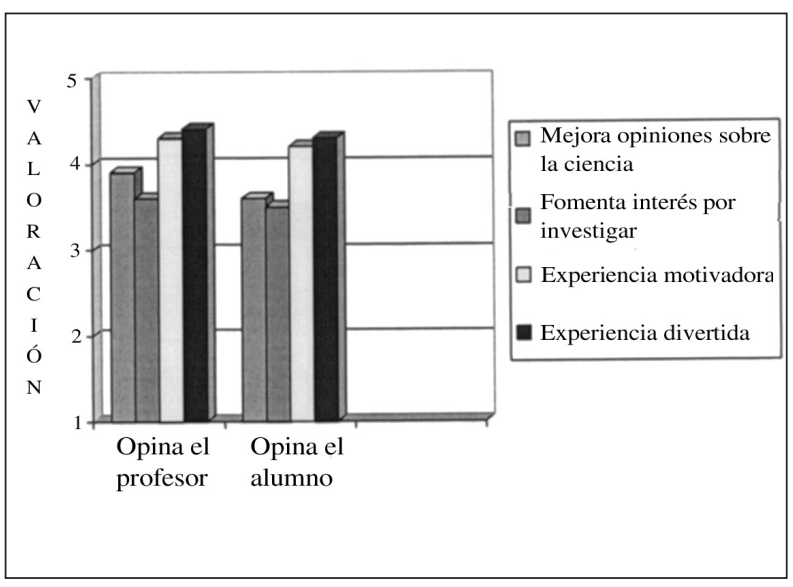

bien en ambos casos pueden considerarse indicadores positivos de la actitud generada. Sobre todo destaca el alto grado de motivación y diversión suscitada, aunque también puede considerarse positiva su repercusión en la mejora de las opiniones sobre la ciencia y en el interés por investigar.

Sin embargo, detectamos una mayor variabilidad en las opiniones sobre la repercusión de «La Ciencia Divertida» en las actitudes hacia el aprendizaje de la ciencia en el aula, lo cual nos hace ser más críticos a la hora de valorar su aportación en este ámbito. En efecto, si bien, en algunos casos, tanto alumnos como profesores reconocieron una incidencia positiva en este terreno, las referencias al respecto fueron más escasas y coexistían con opiniones contrarias:

«Está claro que actividades como éstas sirven para enganchar a los alumnos con las asignaturas de ciencias. En mis alumnos, al menos, pude apreciar un cambio de actitudes notorio en determinados casos.» (Profesora)

«Me ha encantado participar en la experiencia y veo ahora las clases de ciencias de otra manera.» (Alumno de $4^{\circ}$ de ESO)

«Cuando estás con los alumnos en estos ambientes parecen muy motivados por aprender, pero luego viene el día a día en el aula que es mucho más duro.» (Profesor)

«Veo que la ciencia es algo divertido, pero otra cosa es cuando hay que estudiar y todo ese rollo de los exámenes.» (Alumna de $3^{\circ}$ de ESO)

Esta diversidad de opiniones se tradujo, en términos cuantitativos, en puntuaciones intermedias de la escala Likert en uno de los ítems cerrados del cuestionario dirigido a evaluar este aspecto, algo mejor valorado, por cierto, por los profesores que por los alumnos (Fig. 2). Ello supone una cierta indecisión o escepticismo respecto a este punto, lo que en cierto modo indica una pobre repercusión de la experiencia en esta vertiente.

Figura 2

Opiniones de profesores y alumnos sobre la posible incidencia o no de la experiencia en las actitudes en la clase de ciencias.

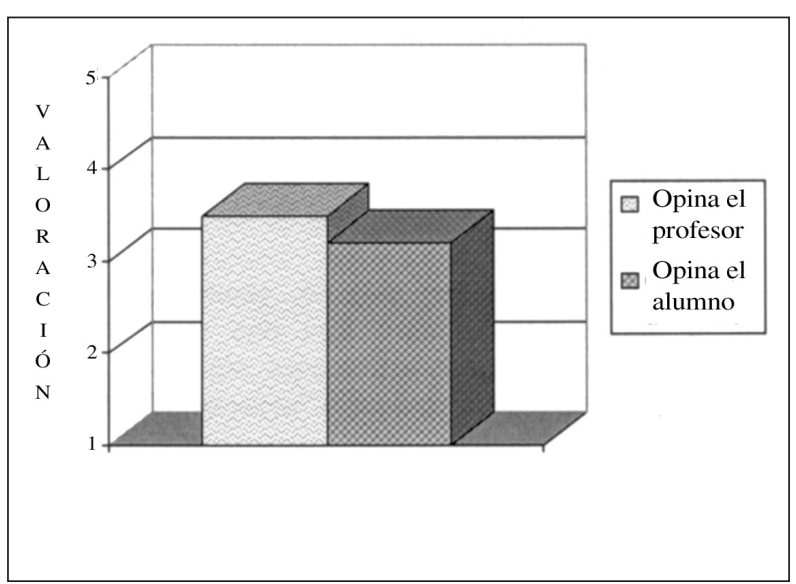


En suma, parece que «La Ciencia Divertida» constituyó una experiencia interesante para los alumnos, al contribuir positivamente a la mejora de sus actitudes hacia las ciencias, pero con una transferencia limitada en el aula. A pesar de ello, el balance global que podemos hacer en este campo es positivo, sobre todo si, a lo dicho, añadimos su contribución en los ámbitos de la autoestima y de la autorrealización del alumnado, aspecto que pasamos a analizar a continuación.

\section{Sobre el nivel de autorrealización promovido}

Otro de los campos bien valorado fue el de la contribución de la experiencia a la realización personal de los alumnos. Así, encontramos referencias al desarrollo de la autoestima promovido en los alumnos a causa del protagonismo que tuvieron en todo el proceso:

«El alumno se ha sentido útil e importante y ha madurado.» (Profesora)

«Los alumnos trabajaban tomando decisiones.» (Profesora)

«Lo más positivo ha sido sentirme protagonista en los experimentos.» (Alumna de $3^{\circ}$ de ESO)

«Lo mejor de todo ha sido para mí convertirme en el centro de atención de la gente que asistió a la exposición.» (Alumna de $4^{\circ}$ de ESO)

En estrecha relación con ello, hemos de destacar también la importancia concedida desde «La Ciencia Divertida» al desarrollo de la autonomía personal del alumno. Se trataba de alentar la capacidad para adoptar iniciativas y tomar decisiones, estimular la actitud creativa y fomentar la responsabilidad y el control sobre el propio aprendizaje. En este sentido, hemos de recordar que, en la experiencia, los alumnos trabajaron con cierta autonomía, aportando ideas, responsabilizándose de su trabajo, manipulando y confeccionando materiales e interaccionando abiertamente con los visitantes durante la fase de exposición al público. Veamos dos referencias al respecto, una de ellas procedente de los comentarios de uno de los grupos de trabajo de profesores y otra de un fragmento de una de las entrevistas finales realizadas con una alumna de $4^{\circ}$ de ESO, la cual ilustra la visión positiva que tuvo el alumnado de la autonomía concedida:

«La evolución de los alumnos [...] ha sido muy positiva, ya que éstos se han encontrado muy a gusto con este trabajo extra. Ellos se han sentido responsables de la exposición, aspecto que considerábamos primordial para el buen funcionamiento del proyecto.» (Grupo de trabajo de profesores núm. 3).

Entrevistador: «¿Qué ha supuesto para ti el participar en "La Ciencia Divertida"?»

Alumna: «Me ha encantado.»

Entrevistador: «¿Sí? ¿Podrías contarme por qué?»

Alumna: «No sé... Todo ha sido muy interesante.»

Entrevistador: «¿Hay alguna cosa que quisieras resaltar?»

Alumna: «No sé [...] Bueno, sí. La verdad es que ha estado muy bien eso de poder participar y aportar ideas [...] y tener que ingeniártelas por tu cuenta para resolver las cosas [...] El profesor nos ayudaba, siempre estaba ahí. Pero luego eras tú la que tenías que buscarte la vida.»
Otro ejemplo del grado de autonomía que propició la experiencia lo encontramos en el hecho de que, en cierta ocasión, fueran los propios alumnos los que, bajo su iniciativa, se encargaran de contactar con algunos museos y centros de ciencias del país con objeto de profundizar en detalles sobre el funcionamiento y construcción de los módulos que elaboraban. Esta actividad fue muy rica para ellos como tarea de búsqueda, de apertura al exterior y de establecimiento de contactos.

Asimismo resultó interesante la experiencia que vivieron los alumnos durante la exposición en público, interaccionando con los visitantes para explicar el fundamento científico del módulo a aquéllos que parecían interesados. Dicho contacto supuso inicialmente, para algunos, un cierto problema por motivos de timidez, pero se acabó resolviendo con la superación del obstáculo, como finalmente reconocían sus profesores y los propios implicados:

«La participación de los alumnos explicando al público la exposición ha contribuido de una forma muy positiva, pues personas que se consideraban tímidas fueron capaces de superar la timidez y llevaron a cabo con brillantez y soltura el trabajo encomendado. Otros alumnos y alumnas más sueltos reafirmaron su personalidad.» (Grupo de trabajo de profesores núm. 4)

«Para mí ha significado mucho el perder la vergüenza de hablar en público.» (Alumna de $3^{\circ}$ de ESO)

«Me ha gustado ver que soy capaz de hablar con soltura explicando cosas a distintas personas.» (Alumna de $4^{\circ}$ de ESO)

Como se desprende de los dos últimos comentarios, la experiencia sirvió también como oportunidad para desarrollar el espíritu de superación ante los obstáculos, algo que también está patente en los dos párrafos que se incluyen a continuación, esta vez referidos a los problemas que surgieron durante el proceso:

«Después de tantos problemas y dificultades, el esfuerzo que realizamos valió la pena.» (Alumno de $4^{\circ}$ de ESO)

«En algunos momentos nos entraron ganas de abandonar cuando vimos las dificultades que surgían [...] Por eso, al final [...] todavía fue mejor ver lo que habíamos conseguido.» (Alumna de $4^{\circ}$ de ESO)

Sin duda, esta capacidad de superación de las dificultades fue posible solamente gracias al buen clima de relaciones que se entabló durante la experiencia entre los alumnos de cada grupo y entre éstos y el profesor correspondiente.

\section{Sobre el clima de convivencia y de relaciones per- sonales desarrolladas}

Una de las mayores contribuciones de «La Ciencia Divertida» fue en el ámbito de la convivencia entre los propios alumnos y entre éstos y el profesor. Parece, por ejemplo, que la experiencia constituyó un excelente escenario para el desarrollo de relaciones humanas entre alumnos y la mejora del trabajo en equipo. 
«Lo mejor ha sido el fomento de las relaciones entre ellos [los alumnos] y el espíritu de equipo.» (Profesor).

«Lo más positivo ha sido haber trabajado en grupo.» (Alumno de $3^{\circ}$ de ESO)

«[Lo mejor ha sido] el contacto con los demás compañeros.» (Alumno de $4^{\circ}$ de ESO).

Por otra parte, la propia naturaleza de la actividad, en la que alumnos y profesores trabajaron sin presión en un ambiente de disciplina compartida, contribuyó notablemente al desarrollo de afectos y buenas relaciones personales entre alumnos y profesores, aspecto que no siempre se da de forma plena dentro de la rutina formal del aula. Por ejemplo, tanto el alumnado como el profesorado participante destacaba la oportunidad que les brindó la experiencia para mejorar la comunicación profesor-alumno:

«El tiempo dedicado a la elaboración de los módulos ha fomentado el diálogo y la convivencia con los alumnos.» (Profesor)

«El trabajo en grupo ha favorecido la colaboración y compañerismo entre alumnos, sin olvidar la relación alumno-profesor.» (Grupo de trabajo de profesores núm. 4)

«Una de las mejores cosas ha sido poder llegar a sentirme más cerca de los profesores.» (Alumna de $4^{\circ}$ de ESO)

Finalmente diremos que, en bastantes ocasiones, tanto el profesorado como el alumnado llegaba a admitir abiertamente que la mejora en el clima de convivencia había afectado positivamente a la dinámica del aula:

«La convivencia entre profesores y alumnos ha mejorado al tratarse de una forma diferente a la habitual, que ha contribuido a mejorar la convivencia en el aula.» (Grupo de trabajo de profesores núm. 2)

«Ahora llego a clase y veo al profesor de otra manera [...] Me parece alguien más cercano.» (Alumno de $3^{\circ}$ de ESO)
Con objeto de dar una visión cuantitativa de las opiniones de profesores y alumnos sobre este tema, hemos incluido la figura 3. En ella se presentan los resultados de dos de los ítems planteados en el cuestionario en formato Likert, los cuales cuestionaban sobre el posible impacto de la actividad en ambas facetas.

Como puede verse, los resultados cuantitativos apoyan los datos cualitativos antes expuestos, indicando de nuevo un impacto positivo de la experiencia en el clima de relaciones personales. Hay que hacer notar que, en términos globales, los profesores valoraron más positivamente dicha incidencia que el alumnado, lo cual sugiere que «La Ciencia Divertida» sirvió no sólo como un instrumento de mejora afectiva para el alumnado sino, sobre todo, para el profesorado.

\section{DISCUSIÓN Y CONCLUSIONES}

Antes de pasar a comentar las conclusiones de esta investigación parece necesario hacer un balance de la confiabilidad de los resultados mostrados. Este tipo de valoraciones, en una investigación cualitativa como la aquí descrita, viene respaldada por criterios de credibilidad y transferibilidad como análogos a los de validez interna y externa de la investigación cuantitativa.

En primer lugar, creemos sinceramente haber dotado a nuestra investigación del rigor metodológico necesario para asegurar, al menos, un mínimo de credibilidad de resultados, característica ésta que, en la investigación cualitativa, suele reconocerse por la observación intensiva y la triangulación, aspectos ambos contemplados en nuestro estudio: el primero de ellos mediante nuestra intervención como observadores participantes, con un acceso rico y continuo a la información procedente de la misma; y el segundo por el uso de instrumentos variados, como han sido el cuestionario, las entrevistas y los informes presentados por los grupos de trabajo de profesores, así como la consiguiente contrastación de los resultados obtenidos de los mismos.

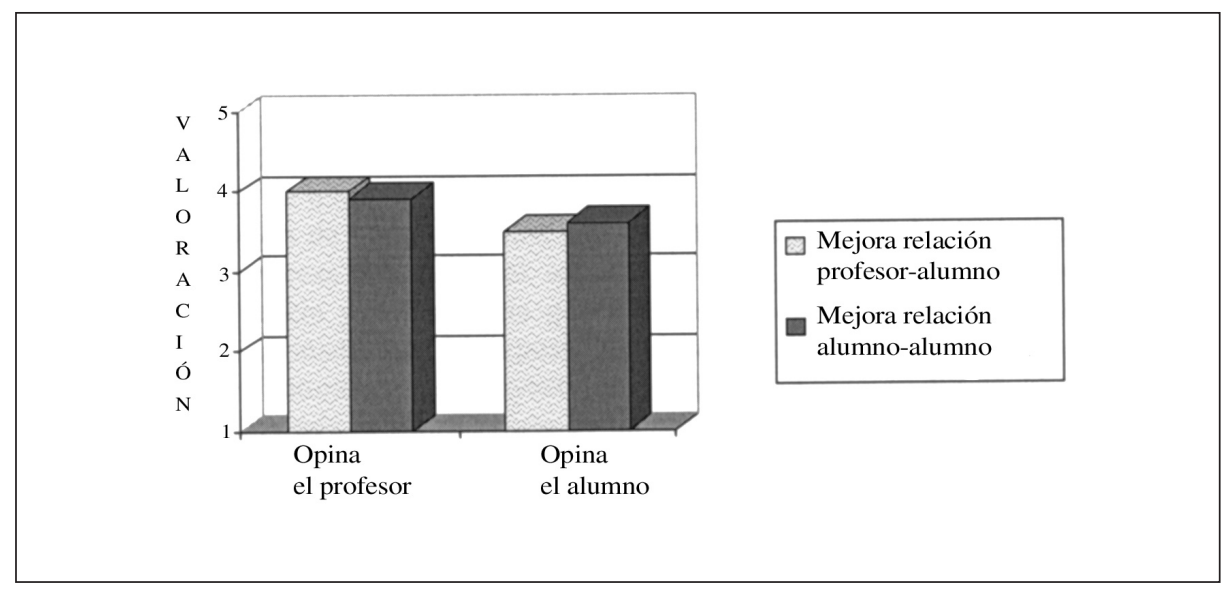


En segundo lugar, hemos de reconocer que un estudio como éste, de carácter marcadamente cualitativo, no permite generalizar sus resultados a cualquier otra población estudiantil. De hecho, la propia voluntariedad del alumnado y el profesorado participante constituye, en sí mismo, un obstáculo importante en este sentido al imponer un cierto sesgo en la muestra encuestada. No obstante, hay que decir que la heterogeneidad del alumnado implicado en la muestra estudiada, así como los resultados positivos obtenidos en la experiencia para algunos alumnos «poco aventajados académicamente» que participaron en ella, nos hacen ser ciertamente optimistas en cuanto a la posibilidad de extrapolar sus conclusiones a otras muestras de alumnos, más o menos semejantes a la nuestra.

Otra cuestión bien distinta es la de averiguar si hay un factor que haya influido más en el éxito de la misma, algo que a nuestro juicio se debió, no a alguno de los elementos per se, sino a la combinación de varios de ellos a la vez, a saber: el carácter experimental e investigativo de la empresa realizada, el sentir como un reto los problemas que se presentaron, la convivencia y camaradería que se fomentó durante el abordaje de esos retos, el sentirse protagonistas en la exposición al público, etc.; factores todos ellos consustanciales con la naturaleza del proyecto global que acometieron y que no pueden analizarse de forma separada al formar parte de un todo. Por ello, lo máximo que podríamos suponer es que actividades similares a ésta, realizadas en una localidad o en un centro escolar, podrían ser de gran interés a la hora de motivar a los alumnos y desarrollar otros factores relacionados con la dimensión afectiva del aprendizaje de las ciencias.

Así pues, podemos concluir que los resultados obtenidos a partir de las distintas fuentes consultadas se muestran bastante prometedores y aportan una visión optimista de la actividad realizada. De forma especial, parece que ésta incidió en la implicación y motivación del alumnado que diseñó los módulos, en su autoestima y autorrealización personal, en la mejora de la convivencia y el clima de relaciones alumno-alumno y alumno-profesor, y en la motivación y satisfacción personal de los alumnos hacia la propia actividad y la ciencia en general.

Sin embargo, no apreciamos grandes logros respecto a la transferencia al aula de las actitudes positivas generadas hacia la experiencia llevada a cabo y hacia la propia ciencia, aunque sí respecto al clima de relaciones personales. Es posible que la eventual transferencia de esas actitudes al aula sea un proceso lento y complejo, y que probablemente requiera una acción más continuada en esta dirección, siempre pensando en plazos más largos de los contemplados en esta evaluación.

La enseñanza de las ciencias en nuestras aulas está monopolizada por el aprendizaje de hechos y conceptos, y, en mucha menor medida, procedimientos de la ciencia. La exclusiva insistencia en contenidos de este tipo, muchas veces áridos y difíciles, hace que el aprendizaje de la ciencia escolar se convierta, para una mayoría de estudiantes, en una experiencia de fracasos, desencuentros y fobia creciente, tal como demuestran numerosos trabajos sobre la imagen de la ciencia (Vázquez y Manassero, 1997). Los aprendizajes del ámbito socioafectivo (actitudes favorables hacia la ciencia, convivencia social positiva, afianzamiento de la motivación y la autoestima, etc.) quedan limitados con frecuencia a las declaraciones de intenciones, sin incidencia práctica en las aulas de ciencias.

En esta situación, la enseñanza de la ciencia se autoexcluye de la participación para conseguir los objetivos más generales de la educación básica, como el desarrollo integral de la persona, donde se incluye el ámbito socioafectivo y la formación para la ciudadanía; pero el fracaso más estridente es su incapacidad para promover la alfabetización científica y tecnológica, una comprensión básica o interés por la ciencia; antes bien, rechaza y discrimina a muchos estudiantes, especialmente las minorías y las mujeres.

Los resultados expuestos de esta experiencia muestran cómo también la enseñanza de las ciencias puede contribuir a alcanzar los objetivos generales de la formación básica de las personas; sobre todo si se prescinde del excesivo culto al cognitivismo, cuya inutilidad ha sido demostrada repetidamente por la extensa investigación sobre las concepciones alternativas. En esta línea de mayor apertura y contribución de la enseñanza de las ciencias hacia la mejor formación general de la ciudadanía se encaminan las reformas de la educación científica de muchos países (AAAS, 1989, 1993; NRC, 1996). Éstas adoptan enfoques didácticos que asumen explícitamente objetivos actitudinales, axiológicos (valores y normas) y de alfabetización científica y tecnológica para todas las personas (Acevedo, 2004), donde el marco del movimiento CTS para la enseñanza de las ciencias parece el más idóneo para lograrlos (Acevedo, Vázquez y Manassero, 2003).

Así mismo, cuando lo que más suele destacarse de la educación secundaria son los problemas de convivencia y disciplina, los resultados obtenidos con esta experiencia sugieren que el desarrollo en los centros escolares de actividades extraacadémicas de este tipo podría ser un revulsivo adecuado para mejorar el clima del aula.

Para terminar, cabe decir que, además de los resultados mostrados en este estudio, parece interesante analizar también en qué medida «La Ciencia Divertida» contribuyó al aprendizaje de los alumnos en el ámbito cognitivo, como también hasta qué punto incidió sobre el profesorado participante. Por ello, actualmente están en preparación sendos artículos, estudiándose en uno de ellos la evolución de los modelos explicativos de los alumnos sobre los fenómenos implicados en los módulos de la exposición, mientras que en el otro se discuten las percepciones de los profesores sobre las aportaciones en su desarrollo profesional. 


\section{REFERENCIAS BIBLIOGRÁFICAS}

AAAS (1989). Science for all americans. Nueva York: Oxford University Press.

AAAS (1990). The liberal art of Science: agenda for action. Washington, DC: American Association for the Advancement of Science.

AAAS (1993). Benchmarks for Science Literacy: A project 2061 report. Nueva York: Oxford University Press.

AAVV (1991). Special issue: Informal sources for learning science. International Journal of Science Education, 13(5).

ACEVEDO, J.A. (1995). Educación tecnológica desde una perspectiva CTS. Una breve revisión del tema. Alambique, 3, pp. 75-84. En línea en Sala de Lecturas CTS+I de la OEI, <http: //www.campus-oei.org/salactsi/acevedo5.htm>, 2001.

ACEVEDO, J.A. (1996). Cambiando la práctica docente en la enseñanza de las ciencias a través de CTS. Borrador, 13, pp. 26-30. En línea en Sala de Lecturas CTS+I de la OEI, <http: //www.campus-oei.org/salactsi/acevedo2.htm>, 2001.

ACEVEDO, J.A. (2004). Reflexiones sobre las finalidades de la enseñanza de las ciencias: educación científica para la ciudadanía. Revista Eureka sobre Enseñanza y Divulgación de las Ciencias, 1(1), pp. 3-16. En línea <http://www.apaceureka.org/revista/Larevista.htm>.

ACEVEDO, J.A. y VÁZQUEZ, A. (2003). Editorial del monográfico «Las relaciones entre ciencia y tecnología en la enseñanza de las ciencias». Revista Electrónica de Enseñanza de las Ciencias, 2(3), <http://www.saum.uvigo.es/reec/>.

ACEVEDO, J.A., VÁZQUEZ, A. y MANASSERO, M.A. (2003). Papel de la educación CTS en una alfabetización científica y tecnológica para todas las personas. Revista Electrónica de Enseñanza de las Ciencias, 2(2), <http: //www.saum.uvigo.es/reec/>.

ACEVEDO-PINEDA, E.B. Ciencia y público. En Sala de Lecturas CTS $+I$ de la $O E I,<$ http://www.campus-oei.org/ salactsi/elsa5.htm $>$.

AUSUBEL, D.P., NOVAK, J.D. y HANESIAN, H. (1989). Psicología educativa. Un punto de vista cognoscitivo. México, DF: Trillas.

BAIRD, J.R. (1997). Perceptions of challenge in science learning. International Journal of Science Education, 19(10), pp. 1195-1209.

BENLLOCH, M. y WILLIAMS, V.N. (1998). Influencia educativa de los padres en una visita al museo de la ciencia: actividad compartida entre padres e hijos frente a un módulo. Enseñanza de las Ciencias, 16(3), pp. 451-460.

CARRASCOSA, J. (1995). Trabajos prácticos de física y química como problemas. Alambique, 5, pp. 67-76.

CRESPO, M. y HERNÁNDEZ, L.M. (2000). Los clubs de ciencia y tecnología como motivación para el trabajo en el área de ciencias: análisis de una experiencia. Actas de los XIX Encuentros de Didáctica de las Ciencias Experimentales, p. 29. Madrid: Facultad de Educación de la Universidad Complutense.

GARRET, R.M. (1995). Resolver problemas en la enseñanza de las ciencias. Alambique, 5, pp. 6-15.

GERBER, B.L., CAVALLO, A.M.L. y MAREK, E.A. (2001). Relationships among informal environments, teaching procedures and scientific reasoning ability. International Journal of Science Education, 23(5), pp. 535-549.

GIL, D. y PAYÁ, J. (1988). Los trabajos prácticos de física y química y la metodología científica. Revista Española de Física, 2(2), pp. 73-79.

GIL, D., CARRASCOSA, J., FURIÓ, C. y MARTÍNEZ-TORREGROSA, J. (1991). La enseñanza de las ciencias en la educación secundaria. Barcelona: ICE-Horsori.

GÓMEZ-CHACÓN, I.M. (1998). Una metodología cualitativa para el estudio de la influencia afectiva en el conocimiento de las matemáticas. Enseñanza de las Ciencias, 16(3), pp. 431-450.

GRIFFIN, J. (1998). Learning science through practical experiences in museums. International Journal of Science Education, 20(6), pp. 655-663.

JONES, L.S. (1997). Opening doors with informal science: exposure and access for our undeserved students. Science Education, 81(6), pp. 663-677.

LAUKENMANN, M., GROB, K. y VAN RHÖNEK, G. (1999). Influence on emotions on classroom learning in physics and German language. Proceedings of second International Conference of the European Science Education Research Association. Kiel, Alemania.

LUCAS, A.M. (1991). «Info-tainment» and informal sources for learning science. International Journal of Science Education, 13(5), pp. 495-504.

LUCAS, A.M., McMNUS, P.M. y THOMAS, G. (1986). Investigating learning from informal sources: listening to conversations and observing play in science. European Journal of Science Education, 8(4), pp. 341-352.

LUCAS, K.B. (2000). One teacher's agenda for a class visit to an interactive science centre. Science Education, 84(2), pp. 524-544.

LYNCH, M.J. y ZENCHAK, J.J. (2002). «Use of Scientific inquiry to explain counterintuitive observations». Comunicación pre-Annual International Conference of the Association for the Education of Teachers in Science. Charlotte, NC, en Rubba, P.A., Rye, J.A., Di Biase, W.J. y Crawford, B.A. (eds.). Proceedings of the 2002 Annual International Conference of the Association for the Education of Teachers in Science, pp. 523-521. Pensacola, FL: AETS.

MAIZTEGUI, A., ACEVEDO, J.A., CAAMAÑO, A., CACHAPUZ, A., CAÑAL, P., CARVALHO, A.M.P., DEL CARMEN, L., DUMAS CARRE, A., GARRITZ, A., GIL, D., GONZÁLEZ, E., GRAS-MARTÍ, A., GUISASOLA, J., LÓPEZ-CEREZO J.A., MACEDO, B., MARTÍNEZTORREGROSA, J., MORENO, A., PRAIA, J., RUEDA, C., TRICARICO, H., VALDÉS, P. y VILCHES, A. (2002). Papel de la tecnología en la educación científica: una dimensión olvidada. Revista Iberoamericana de Educación. 28, pp. 129-155. En línea <http://www.campus-oei.org/ revista/rie28a05.PDF>.

MANASSERO, M.A. y VÁZQUEZ, A. (1998). Validación de una escala de motivación de logro basada en la teoría atribucional de Weiner. Psicothema, 10, pp. 333-351.

MANASSERO, M.A. y VÁZQUEZ, A. (2001). Análisis empírico de dos escalas de motivación escolar. Revista Española de Motivación y Emoción, 2, pp. 37-58.

MATOS, J., OLIVA, J.M., BONAT, M., BUENO, E., DÍEZ, C., DOMINGUEZ, J. y OSUNA, J. (2001). «Las exposiciones científicas como instrumento de motivación e implicación del alumnado y del profesorado de ciencias». Comunicación presentada en el VI Congreso Internacional sobre Investigación en la Didáctica de las Ciencias, Barcelona. Enseñanza de las Ciencias, núm. extra, pp. 269-270. 
McCLELLAND, D.C. (1961). The achieving society. Princeton, NJ: Van Nostrand.

McLEOD, D.B. (1990). Information processing theories and mathematics learning: the role of affect. International Journal of Educational Research, 14, pp. 13-29.

MEDVED, M.I. y OATLEY, K. (2000). Memories and scientific literacy: remembering exhibits from a science centre. International Journal of Science Education, 22(10), pp. 1117-1132.

MEREDITH, J.E., FORTNER, R.W. y MULLINS, G.W. (1997). Model of affective learning for nonformal science education facilities. Journal of Research in Science Teaching, 34(8), pp. 805-818.

NRC (1996). National Science Education Standards. Washington, DC: National Academic Press.

OLIVA, J.M. y MATOS, J. (1999a). La ciencia recreativa como recurso para la enseñanza de las ciencias y el desarrollo profesional docente. Perspectiva CEP, 1, pp. 89-101.

OLIVA, J.M. y MATOS, J. (1999b). La ciencia recreativa como centro de interés para el desarrollo profesional de profesores de ciencias en activo. Actas del IX Congreso INFAD 2000. Infancia y Adolescencia, vol. II, pp. 851-855. Universidad de Cádiz.

OLIVA, J.M. y MATOS, J. (2000). Sobre las relaciones entre la didáctica de las ciencias y la comunicación social de la ciencia, en Páramo, E. (coord.). Comunicar la Ciencia en el siglo XXI, pp. 338-341. Granada: Proyecto Sur de Ediciones.

OLIVA, J.M., MATOS, J., BUENO, E., DÍEZ, C., DOMÍNGUEZ, J., OSUNA, J. y BONAT, M. (2002). Las exposiciones científicas escolares y su contribución al ámbito afectivo en los alumnos participantes. Actas de los XX Encuentros de Didáctica de las Ciencias Experimentales, pp. 521-529. La Laguna.

PINTRICH, P.R., MARX, R.W. y BOYLE, R.A. (1993). Beyond cold conceptual change: the role of motivational beliefs and classroom contextual factors in the process of conceptual change. Review of Educational Research, 63(2), pp. 167-199.

POSNER, G., STRIKE, K., HEWSON, P. y GERTZOG, W. (1982). Accommodation of a scientific conception: Toward a theory of conceptual change. Science Education, 66(2), pp. 211-227.

POZO, J.I. y GÓMEZ-CRESPO, M.A. (1997). ¿Qué es lo que hace difícil la comprensión de la ciencia? Algunas explicaciones y propuestas para la enseñanza, en Del Carmen, L. (coord.). La enseñanza y el aprendizaje de las ciencias de la naturaleza en la educación secundaria, pp. 73-105. Barcelona: ICE-Horsori.

RAMSDEN, J.M. (1998). Mission impossible?: can anything be done about attitudes to science. International Journal of Science Education, 20(2), pp. 125-137.

REID, D.J. y HODSON, D. (1989): Science for all. Londres: Casell. Trad. de Martín-Díaz, M.J. y García-Lucía, L.A. (1993). Ciencia para todos en secundaria. Madrid: Narcea.

RIX, C. y McSORLEY, J. (1999). An investigation into the role that school-based interactive science centres may play in the education of primary-aged children. International Journal of Science Education, 21(6), pp. 577-593.
RODRÍGUEZ, G., GIL, J. y GARCÍA, E. (1996). Metodología de la investigación cualitativa. Málaga: Aljibe.

RUSSELL, I. (1990). Visiting a science centre: what's on offer? Physics Education, 25, pp. 258-262.

SCHIBECI, R.A. y RILEY, J.P. (1986). Influence of students' background and perceptions on science attitudes and achievement. Journal of Research in Science Teaching, 23(3), pp. 177-187.

SEMPER, R.J. (1990). Science museums as environments for learning. Physics Today, 43, pp. 2-8.

SÈRÉ, M.G. (2002). La enseñanza en el laboratorio. ¿Qué podemos aprender en términos de conocimiento práctico y de actitudes hacia la ciencia? Enseñanza de las Ciencias, 20(3), pp. 357-368.

STEVENSON, J. (1991). The long-term impact of interactive exhibits. International Journal of Science Education, 13(5), pp. 521-531.

STRIKE, K.A. y POSNER, G.J. (1992). A revisionist theory of conceptual change, en Duschl, R. y Hamilton, R. (eds.). Philosophy of science, cognitive psychology and educational theory and practice, pp. 47-176. Albany, Nueva York: SUNY.

TAMIR, P. (1990). Factors associated with the relationship between formal, informal and nonformal science learning. Journal of Environmental Education, 22(1), pp. 34-42.

VALDÉS, P., VALDÉS, R., GUISASOLA, J. y SANTOS, T. (2002). Implicaciones de las relaciones ciencia-tecnología en la educación científica. Revista Iberoamericana de Educación, 28, pp. 101-128. En línea <http://www.campusoei.org/revista/rie28a04.PDF>.

VÁZQUEZ, A. y MANASSERO, M.A. (1995). Actitudes relacionadas con la ciencia: una revisión conceptual. Enseñanza de las Ciencias, 13(3), pp. 337-346.

VÁZQUEZ, A. y MANASSERO, M.A. (1997). Escribir sobre ciencia: imagen de la ciencia y los científicos. Comunicación, Lenguaje y educación, 6-7, pp. 181-206.

Von SECKER, C.E. y LISSITZ, R.W. (1999). Estimating the impact of instructional practices on student achievement in science. Journal or Research in Science Teaching, 36, pp. 1110-1126.

WATTS, M. y ALSOP, S. (2000). The affective dimensions of learning science. International Journal of Science Education, 22(2), pp. 1219-1220.

WEINER, B. (1992). Human motivation. Metaphors, Theories and Research. Newbury Park: SAGE.

WELLINGTON, J. (1991). Newspaper science, school science: friends or enemies? International Journal of Science Education, 13(4), pp. 363-372.

WHEATLEY, G.H. (1991). Constructivist perspectives on science and mathematics learning. Science Education, 75(1), pp. 9-21.

YAGER, B.E. y YAGER, S.O. (1985). Changes in perceptions of science for third, seventh and eleventh grade students. Journal of Research in Science Teaching, 22(4), pp. 347-358. 


\section{ANEXO}

Algunos ítems procedentes del cuestionario presentado a los profesores

Valora cómo crees que ha repercutido en los alumnos su participación en «La Ciencia Divertida». Para ello marca en cada caso con una cruz. la opción que creas más oportuna.

Item 4. Ha contribuido a mejorar sus opiniones sobre la ciencia.

Nada $\square \quad$ Poco $\square \quad$ Regular $\square \quad$ Bastante $\square \quad$ Totalmente

Ítem 5. Ha mejorado sus actitudes en las clases de ciencias.
Nada
Poco
Regular
Bastante
Totalmente

Ítem 9. Ha contribuido a la mejora de la convivencia con el profesor.
Nada
Poco
Regular
Bastante
Totalmente 